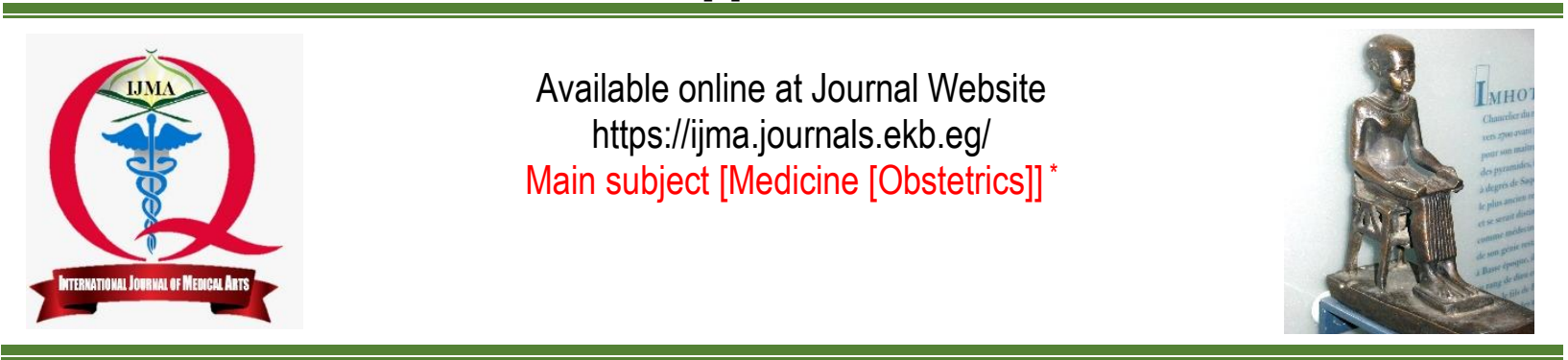

Original article

\title{
Ultrasound in Prediction of Threatened Abortion in Early Pregnancy: A clinical study
}

\author{
Shimaa Shaker Zeed Saleh ${ }^{\mathbf{a}}$; Khattab Abd El-halim Omar Khattab ${ }^{\mathbf{b}}$; Ehab Mohammed Elhelww
}

Department of Obstetrics and Gynecology, Damietta Specialized Hospital, Ministry of Health, Egypt ${ }^{[a]}$.

Department of Obstetrics and Gynecology, Damietta Faculty of Medicine, Al-Azhar University, Egypt ${ }^{[\mathrm{b}]}$.

Corresponding author: Shimaa Shaker Zeed Saleh

Email: a drss37330@gmail.com

Submitted at: September 25, 2019; Revised at: April 14, 2020; Accepted at: April 15, 2020; Available online at: April 15, 2020

DOI: 10.21608/ijma.2020.17353.1032

\section{ABSTRACT}

Background: Early pregnancy loss is a challenging health problem and the prediction of exposed females is mandatory to permit early intervention and prevention.

Aim of the work: To assess utility of ultrasound in detection of threatened abortion in early pregnancy.

Patients and Methods: One-hundred females with history of threatened abortion were included. A written consent was obtained from each participant. Patients were divided into two groups: Group [l]: 85 Cases who continued their pregnancy. Group [II]: 15 Cases ended by abortion. All females were submitted to: detailed history, clinical examination [General and abdominal] and investigations in the form of ultrasound. Data were collected and statistically analyze.

Results: $15 \%$ of studied females had early miscarriage. There was a significant relation between occurrence of abortion and gestational age as abortion was more frequent with reduced gestational age. In addition, high parity [P1-2] was significantly associated with abortion. Also, current abortion associated with abortion in previous pregnancy, subchorionic hematoma, irregular wall of gestational sac and low fetal heart rate. The best cutoff of fetal heart rate is 100 beat/minute. The sensitivity of fetal heart rate in prediction of abortion was $67.1 \%$, specificity was $80.0 \%$, PPV was $95.0 \%$, NPV was $30.0 \%$ and overall accuracy was $69 \%$.

Conclusion: The sonographic findings have a prognostic value that interacts with other clinical and maternal factors analyzed. Some parameters considered as prognostic factors and include irregular wall of gestational sac, perigestational hemorrhage and embryonic bradycardia.

Keywords: Ultrasound; Miscarriage; First trimester; Gestational sac; Perigestational hematoma.

This is an open access article under the Creative Commons license [CC BY] [https://creativecommons.org/licenses/by/2.0/]

Please cite this article as: Saleh SSZ, Khattab KAO, Elhelw EM. Ultrasound in prediction of threatened abortion in early pregnancy: A clinical study. IJMA 2020; 2[2]: 451-456.

* Main subject and any subcategories have been classified according to researchers' main field of study. 


\section{INTRODUCTION}

Loss of pregnancy is a stressful condition for both the physician and patient. Not all pregnancies ended with live birth of an infant and most of conception losses are not diagnosed. Threatened miscarriage was recognized as any bleeding in the first half of pregnancy. It was reported in $20-25 \%$ of all pregnancies. Thus, it considered a common complication of pregnancy[1].

Ultrasound has had its profound influence on the practice of medicine, either in obstetrics or in gynecology. The invention of transvaginal ultrasound has markedly enhanced the assessment of early pregnancy in cases of threatened abortion. The rate of fetal loos is about $15 \%$ in females with threatened abortion and a living baby in first trimester ${ }^{[2]}$.

Hanchate[3] assessed utility of ultrasound in detection of threatened abortion. They concluded that ultrasonography is useful in detection of pregnancy complication. The use of color Doppler is beneficial in assessing abnormalities. Ultrasound checkup has become the "gold standard" in assessment and follow-up of pregnancy and the development of complications in early pregnancy. Ultrasonographic findings such as irregular wall of gestational sac, low fetal heart rate [intrauterine bradycardia], perigestational hematoma etc. are indicative of threatened abortion. It has helped in managing the patients.

\section{AIM OF THE WORK}

The aim of the present study is to assess utility of ultrasound in detection of threatened abortion in early pregnancy.

\section{PATIENTS AND METHODS}

This a prospective, cross sectional study, which included one hundred pregnant women with history of threatened abortion [vaginal bleeding with or without pain and sonographic seen gestational sac with pulsating fetal pole] recruited from Obstetrics and Gynecology Clinic, Al-Azhar University [Damietta[. The study duration extended between January and August 2019. After explanation of the nature of the study, a verbal and written consent was obtained from each participant before participation and the study was approved by the institutional review board of Damietta Faculty of Medicine, AlAzhar University.
The inclusion criteria were: 1] Pregnant women with threatened abortion, and 2] age ranged from 20 to 35 years. On the other side, exclusion criteria were: 1] All cases of recurrent spontaneous miscarriage were excluded; 2] Patients with multiple pregnancies, molar pregnancy, ectopic pregnancy; 3] Missed or incomplete abortion; 4] Maternal history of systemic diseases that are known to increase risk of abortion such as systemic lupus erythematosus; and 5] Maternal history of uterine anatomic abnormalities.

Patients in the study were divided into two groups: Group [l]: 85 Cases with continued pregnancy [without abortion]. Group [II]: 15 Cases ended by abortion.

After selection, counseling, explaining the procedure to all participants, and obtaining a written consent to participate in the study; all participants were submitted to: detailed history [personal, menstrual, obstetrics, past and family history], clinical examination [General and abdominal] and investigations in the form of ultrasound.

Ultrasonography: ultrasound scan was performed using a Voluson 730Pro machine [GE, Milan, Italy] equipped with a multifrequency volume endovaginal probe. Patients were scanned at twoweek intervals from 6 to 20 weeks. Ultrasound assessment with 5- $8 \mathrm{MHz}$ transducer included: normal Fetal Heart Rate [FHR] ranged between 90 and 160 beats per minute ${ }^{[4]}$; Number of fetuses; Gestational age [calculated from the crown-rump length together with the gestational sac diameter at the first examination]; Cardiac activity, placental separation or hematoma if present; with the transvaginal probe placed close to the uterus, with particularly no intervening bowel and bladder was empty. The vaginal probe was covered with an examining glove containing a small amount of gel. The gel ensured good contact between the transducer and the covering glove. Care was exerted to avoid trapping of any air bubbles, which might have created unwanted artifacts on the screen. Cross infection was prevented by the usage of probe cover and disinfectants. Transvaginal probe was introduced into the vagina and positioned in the anterior fornix, and the internal \& external cervical os are identified.

Statistical analysis of data: The data was analyzed by statistical package for social sciences 
[SPSS] version 19 [SPSS Inc, Chicago, USA]. Categorical data expressed as frequency and percent, while quantitative data expressed as mean \pm standard deviation [SD], minimum and maximum. Student " $\mathrm{t}$ " test or Chi squares tests used for comparison between two groups [quantitative and qualitative respectively]. $p$ value $\leq 0.05$ were considered significant.

\section{RESULTS}

The present prospective study included 100 women within the reproductive age; 85 of them continued pregnancy and 15 of them ended by abortion. In the present study, age ranged from 20 to 35 years with a mean of $27.5 \pm 4$ years, gestational sac $10 \pm 1.5$, duration from last pregnancy till current pregnancy $4.56 \pm 0.78$ years, and fetal heart rate $120 \pm 26.5 \mathrm{bpm}$ [Table 1]. Results of the present work revealed that, there was a significant relation between occurrence of abortion and gestational age [p < 0.05] as abortion was more frequent with reduced gestational age. In addition, high parity [P12] was significantly associated with abortion. Also, current abortion also associated with abortion of previous pregnancy, sub-chorionic hematoma, irregular wall of gestational sac and low fetal heart rate. On the other side, maternal age, previous abortion, pelvic pain, duration between last pregnancy till current pregnancy were not associated with current abortion [Table 2]. No significant correlation was found between fetal heart rate and gestational age $[r=-0.15]$.

Regarding fetal heart rate, the best cutoff is 100. The sensitivity of FHR in prediction of abortion was $67.1 \%$, specificity was $80.0 \%$, PPV was $95.0 \%$, NPV was $30.0 \%$ and overall accuracy was $69 \%$ [Table 3].

Table [1]: Descriptive data of studied females

\begin{tabular}{|c|c|c|}
\hline \multirow{2}{*}{\multicolumn{2}{|c|}{ Age [years] }} & Statistics $[n=100]$ \\
\hline & & $27.5 \pm 4 ; 20-35$ \\
\hline \multicolumn{2}{|c|}{ Gestational age at inclusion } & $10 \pm 1.5 ; 5-14$ \\
\hline \multicolumn{2}{|c|}{ Parity [P0, P1-2, P3-4] } & $10,52,38$ \\
\hline \multicolumn{2}{|c|}{ Previous abortions $[0,1,2]$} & $62,23,15$ \\
\hline \multicolumn{2}{|c|}{ Duration from last pregnancy till current pregnancy/years } & $4.56 \pm 0.78 ; 1.5-8$ \\
\hline \multirow[t]{3}{*}{ Last pregnancy fate } & Delivery & $87[87 \%]$ \\
\hline & Abortion & $3[3.0 \%]$ \\
\hline & Primigravida & $10[10.0 \%]$ \\
\hline \multirow[t]{2}{*}{ Gestational sac wall } & Regular & $49[49 \%]$ \\
\hline & Irregular & $51[51 \%]$ \\
\hline \multicolumn{2}{|l|}{ Pelvic pain } & $85[85 \%]$ \\
\hline \multicolumn{2}{|l|}{ FHR } & $120 \pm 26.5 ; 70-155$ \\
\hline \multirow[t]{2}{*}{ Pregnancy outcome } & No abortion & $85[85 \%]$ \\
\hline & Abortion & $15[15 \%]$ \\
\hline
\end{tabular}

Table [2]: Relation between maternal age and gestational age and occurrence of abortion

\begin{tabular}{|c|c|c|c|c|c|}
\hline \multirow{2}{*}{\multicolumn{2}{|c|}{ Maternal age [vears] }} & No abortion [ $n=85]$ & Abortion [n = 15] & Test & $\mathbf{P}$ \\
\hline & & $27.5 \pm 5$ & $26.5 \pm 2.5$ & 0.5 & $0.4[\mathrm{NS}]$ \\
\hline \multicolumn{2}{|l|}{ Gestational age } & $10 \pm 1.5$ & $9 \pm 0.5$ & 2.99 & $0.04[S]$ \\
\hline \multirow{3}{*}{ Parity } & PO & $10[11.8 \%]$ & $0[0.0 \%]$ & \multirow{3}{*}{16.29} & \multirow{3}{*}{$<0.001$} \\
\hline & P1-2 & $37[43.53 \%]$ & $15[100.0 \%]$ & & \\
\hline & P3-4 & $38[44.7 \%]$ & $0[0.0 \%]$ & & \\
\hline \multirow{3}{*}{ Previous abortion } & None & $50[58.8 \%]$ & $12[80.0 \%]$ & \multirow{3}{*}{5.27} & \multirow{3}{*}{$0.07[\mathrm{NS}]$} \\
\hline & Once & $23[27.1 \%]$ & $0[0.0 \%]$ & & \\
\hline & Twice & $12[14.1 \%]$ & $3[20.0 \%]$ & & \\
\hline \multicolumn{2}{|c|}{ Pelvic pain } & $70[82.4 \%]$ & $15[100.0 \%]$ & 3.11 & $0.08[\mathrm{NS}]$ \\
\hline \multicolumn{2}{|c|}{ Duration from last pregnancy } & $3.99 \pm 0.28$ & $4.12 \pm 0.20$ & 1.72 & $0.09[\mathrm{NS}]$ \\
\hline \multirow{3}{*}{$\begin{array}{l}\text { Outcome of last } \\
\text { pregnancy }\end{array}$} & Delivery & $74[87.1 \%]$ & $13[86.7 \%]$ & \multirow{3}{*}{8.01} & \multirow{3}{*}{$0.02[S]$} \\
\hline & Abortion & $1[1.2 \%]$ & $2[13.3 \%]$ & & \\
\hline & Primigravida & $10[11.8 \%]$ & $0[0.0 \%]$ & & \\
\hline \multicolumn{2}{|l|}{ Sub-chorionic hematoma } & $3[3.53 \%]$ & $3[20.0 \%]$ & 6.13 & $0.013[S]$ \\
\hline \multirow{2}{*}{ Wall of gestational sac } & Irregular & $38[44.7 \%]$ & $13[86.7 \%]$ & \multirow{2}{*}{8.98} & \multirow{2}{*}{$0.003[S]$} \\
\hline & Regular & $47[55.3 \%]$ & $2[13.3 \%]$ & & \\
\hline \multicolumn{2}{|l|}{ FHR } & $124 \pm 25$ & $101.5 \pm 34.5$ & 2.5 & $0.003[S]$ \\
\hline
\end{tabular}

FHR: Fetal heart rate 
Table [3]: Role of FHR in prediction of occurrence of abortion

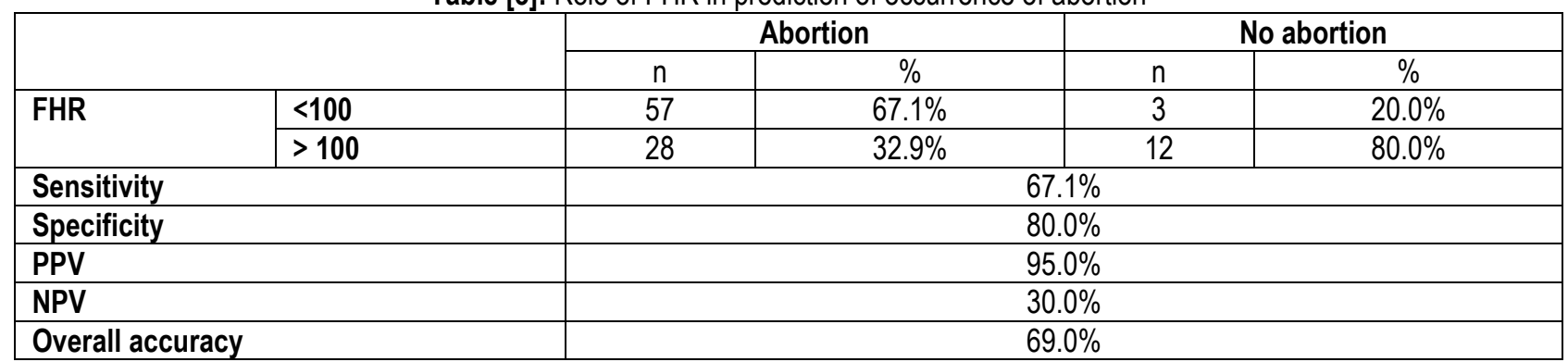

FHR: fetal heart rate; PPV: positive predictive value, NPV: negative predictive value.

\section{DISUCSSION}

In our study, $15 \%$ ended in abortion, which increased in early than late gestational age. Cunningham et al. ${ }^{[5]}$ reported that, up to $15 \%$ of pregnancies could end with miscarriage; and about fourth of females experience abortion in their lifetime, and about $80.0 \%$ of miscarriages occurs early in pregnancy [in the first trimester] and the rate decreased with advancement of gestational age.

Results of the present work are in accordance with Salim et al.[6] who reported that, earl miscarriage is linked to decreased gestational age.

Abortion of the current pregnancy is associated with higher abortions in last pregnancy [13.3\% vs 1.2\%]. Bhattacharya and Bhattacharya[7] found a higher risk of abortion and complicated pregnancy among females with one prior miscarriage. Gunnarsdottir et al. ${ }^{[8]}$ confirmed that females with a history of more than two abortions were exposed to an increased risk of complicated pregnancy. In addition, Benton et al.[9] explained higher rate of complicated pregnancies to placental dysfunction caused by placental ischemia due to implantation insufficiency during the first trimester with consequent abortion.

In accordance with results of the present work, Hamela-Olkowska et al.[10] reported increased pelvic pain in patients with abortion when compared to those without abortion [74 vs 16 patients respectively]. Hanchate ${ }^{[3]}$ assessed usage of ultrasound in prediction of threatened abortion. They revealed that, maternal age was not associated with abortion, and pelvic pain was incrased with abortion. However, the difference was statistically nonsignificant.

In our study, there was statistically significant relation between occurrence of abortion and fetal heart rate, where reduced heart rate was associated with increased abortion. These results are in agreement with Doubilet and Benson[11]. Comparable results were reported by HamelaOlkowska et al.[10] who concluded that the mean embryonic heart rates during the $6^{\text {th }}-7^{\text {th }}$ week of pregnancy are lower when compared with the other gestational ages. Oztekin ${ }^{[12]}$ concluded that a heart rate lower than $100 \mathrm{bpm}$ will indicate a poor prognosis irrespective of gestational age. Al Mohamady et al. ${ }^{[13]}$ found that the mean FHR was $156.9 \pm 20$ bpm for the continued group and $122 \pm 9$ for the aborted group, with statistically significant difference $[P<0.001]$. Furthermore, Hanchate ${ }^{[3]}$ showed that group I [Aborted] had mean fetal heart 79.2 beats/min whereas group II [continued] had 125.2 beats/ min. The difference was statistically significant. Romero-Gutiérrez et al.[14] determined which blood flow measurements are associated with poor outcome in these women. They concluded that altered fetal heart rate, assessed with Doppler ultrasonography, are associated with adverse outcome in women with threatened abortion.

Our study showed that there is a significant difference between the 2 groups in the incidence of subchorionic hematoma [SCH] as $3.53 \%$ in the cases without abortion and $20 \%$ in cases with abortion. Benavides-Reyes et al.[15] reported increased rate of abortion with $\mathrm{SCH}$ and concluded that, there is an association between $\mathrm{SCH}$ volume and abortion in first trimester. Saraswat et al.[16] reported that, $\mathrm{SCH}$ usually arises from the placenta. The mechanisms explaining associated comorbidities may rely on the interference between the chorionic membrane and the uterine wall

Regarding fetal heart rate, the best cutoff is 100 with sensitivity of $67.1 \%$ and specificity of $80 \%$. Abdel Wahab et al.[17] could not find any cutoff values that could predict the occurrence of 
miscarriage. This may be attributed to small sample size and short period of follow up.

In the present study, there were statistical significance association between gestational sacs wall and abortion incidence, and high prevalence of abortion with irregular sacs wall. Berdahl et al.[18] reported that, abnormal sonographic appearance of sac is linked to poor outcome of gestation; therefore, the sonographic data about the sac could be used in prediction of pregnancy outcome [if pregnancy will end up in abortion]. Thus, it has been suggested that abnormal manifestations [e.g., large size, asymmetrical shape, and the presence of echogenic material [e.g., calcification] within the yolk sac, may indicate an increased risk of spontaneous abortion.

Tan et al.[19] [2011] reported that $17 \%$ of gestations [31 of 183] had sacs with an abnormal shape of the wall. Sacs with an abnormal [bizarre] shape of the wall were recognized in $21.8 \%$ of the 6 week-old gestations [17 of 78], $18.2 \%$ of the 7 -weekold gestations [ 12 of 66 ], and $5.1 \%$ of the 8 -week-old gestations [2 of 39]. There was a tendency of decreased irregular sacs rate with advancement of gestational age. However, the difference was not statistically significant. Spontaneous miscarriage recognized in 6 of the 183 [3.3\%]. Four of the gestations ended by spontaneous abortion were 6 weeks old, one was 7 weeks and one was 8 weeks old. An irregular shape of the sac was recognized in only one of 6 pregnancies that had spontaneous abortion, which was 6 weeks old at the time of abortion. Additionally, the rates of spontaneous miscarriages were found to be statistically comparable for pregnancies with regular and irregular sac shapes.

Abdel Wahab et al.[17] concluded that the existence of good uterine blood supply is a vital requirement for implantation to be successful and for continuation of pregnancy. This was confirmed by high resistance of uterine artery blood flow and decreased endometrial blood flow in recurrent abortions and patients with unexplained recurrent pregnancy loss [RPL] may have aberrations in the uterine and endometrial blood supply.

Hanchate ${ }^{[3]}$ concluded that ultrasonography is useful in detection of pregnancy complication. The use of color Doppler is beneficial in assessing abnormalities. Ultrasound examination is considered the "gold standard" in follow-up of early pregnancy and its outcome. Findings such as the irregular wall of the sac, reduced fetal heart rate [bradycardia], perigestational hemorrhage etc. are suggestive of threatened miscarriage. It has helped in the management of patients.

Also, Bhoil et al.[20] concluded that, color doppler of spiral artery is a useful indicator in prediction of threatened abortion.

In conclusion, the sonographic data of traditional ultrasound have been evaluated and these have a prognostic value, besides other clinical and maternal factors. Some other variables obtained by conventional ultrasound could be considered as prognostic factors [irregular wall of gestational sac, perigestational hemorrhage, embryonic bradycardia etc].

\section{Financial and Non-Financial Relationships and Activities of Interest}

None

\section{REFERENCES}

1. Ayaty S, Roudsari FV, Tavassoly F. CA-125 in normal pregnancy and threatened abortion. Iranian J Reprod Med 2007; 5[2]: 57-60.

2. Predanic M, Chervenak FA, Reece EA. Basic Principles of Ultrasound. Chapter 19, In: Handbook of Clinical Obstetrics: The Fetus and Mother, E. Albert Reece MD, PhD, MBA John C. Hobbins MD Norman F. Gant Jr. MD [eds], Third edition 2007; pp 117-129. [10.1002/ 9780470753323.ch19].

3. Hanchate V. Ultrasound in prediction of threatened abortion in early pregnancy: A clinical study. Int J Med Health Res. 2017; 3[3]: 79-81

4. Papaioannou GI, Syngelaki A, Maiz N, Ross JA , Nicolaides KH. Ultrasonographic prediction of early miscarriage. Hum Reprod. 2011; 26: 1685-1692. [DOI: 10.1093/ humrep/ der130].

5. Cunningham FG, Leveno KJ, Bloom SL. Abortion. In: Williams Obstetrics [23rd edition], Publ. McGraw Hill [U.S.A] 2010; 215-234.

6. Salim A, Zalud I, Farmakides G, Schulman H, Kurjak A, Latin V. Corpus luteum blood flow in normal and abnormal early pregnancy: Evaluation with transvaginal color and pulsed Doppler sonography. J Ultrasound Med 1994; 13 [12]: 971-975. [DOI:10.7863/jum.1994.13. 12. 971].

7. Bhattacharya $\mathbf{S}$, Bhattacharya $\mathbf{S}$. Effect of miscarriage on future pregnancies. Women's Health [Lond Engl] 2009; 5:5-8. [DOI:10.2217/17455057.5.1.5].

8. Gunnarsdottir J, Stephansson 0 , Cnattingius $S$, Åkerud $\mathrm{H}$, Wikström AK. Risk of placental dysfunction 
disorders after prior miscarriages: a population-based study. Am J Obstet Gynecol 2014; 211[1]: 34.31-38. [DOI:10.1016/j.ajog.2014.01.041].

9. Benton J, McCowan LM, Heazell AE, Grynspan D, Hutcheon JA, Senger C, et al. Placental growth factor as a marker of fetal growth restriction caused by placental dysfunction. Placenta 2016; 42: 1-8. [DOl:10.1016/ j.placenta.2016.03.010].

10. Hamela-Olkowska A, Wiech K, Jalinik K, Zarviewski D, Kornatowski L, Dangel J. Evaluation of the embryonic and foetal heart rate at $6[+0]$ to $11[+6]$ weeks of gestation. Ginekol Pol 2009; 80: 188-192 [English Abstract]. [PMID:19382610].

11. Doubilet PM, Benson CB. Outcome of first-trimester pregnancies with slow embryonic heart rate at 6-7 weeks gestation and normal heart rate by 8 weeks at US. Radiology 2005; 236[2]: 643-646. [DOI:10.1148/ radiol. 2362040880].

12. Oztekin O. First trimester ultrasound: Current approaches and practical pitfalls. J Med Ultrason [2001]. 2009; 36 [4]: 161-75. [DOI: 10.1007/s10396-009-0226-2].

13. Al Mohamady M, Abdel Fattah Gh, Elkattan E. Correlation of serum CA-125 and progesterone levels with ultrasound markers in the prediction of pregnancy outcome in threatened miscarriage. Int J Fertil Steril. 2016; 9[4]: 506-511. [DOI: 10.22074/ ijfs.2015.4609].

14. Romero-Gutiérrez G, Huebe-Martinez AA, AmaralNavarro I, Ruiz-Trevio AS. Doppler ultrasound assessment in women with threatened abortion. Clin Med Res. 2013; 2[3]: 24-28. [DOI: 10.11648/j.cmr. 20130203. 11].
15. Benavides-Reyes I, Reyna-Villasmil E, Mejia-Montilla J, Torres-Cepeda D, Navarro-Briceño Y, SantosBolívar J, et al. Subchorionic hematoma volume in the first trimester and risk of spontaneous abortion. Revista Latinoamericana de Hipertensión 2015; 10[3]: 56-60.

16. Saraswat L, Bhattacharya S, Maheshwari A, Bhattacharya S. Maternal and perinatal outcome in women with threatened miscarriage in the first trimester: a systematic review. BJOG 2010; 117:245-57. [DOI:10.1111/j.1471-0528.2009. 02427.x]

17. Abdel Wahab H, Salah E, Zain E, Abdelgany M, Youssef MA. Uterine artery Doppler and subendometrial blood flow in patients with unexplained recurrent miscarriage. Middle East Fertil Society J 2011; 16: 209_ 214. [DOI: 10.1016/j.mefs.2011.04.001].

18. Berdahl DM, Blaine J, Van Voorhis B, Dokras A. Detection of enlarged yolk sac on early ultrasound is associated with adverse pregnancy outcomes. Fertil Steril 2010; 94:1535-1537. [DOI: 10.1016/j.fertnstert.2009. 12.064].

19. Tan S, Ali I, Mine K, Meral A, Arifoğlu M, Teber MA, Karaoğlanoğlu M. Irregular Yolk SacShapels It Really Associated with an Increased Risk of Spontaneous Abortion? J Ultrasound Med 2011; 30:31-36 [DOI: 10. 7863/ jum.2011.30.1.31].

20. Bhoil R, Kaushal S, Sharma R, Kaur J, Sharma T, Thakur R, Sharma R. Color Doppler ultrasound of spiral artery blood flow in mid first trimester [4-8 weeks] in cases of threatened abortion and in normal pregnancies. J Ultrason. 2019;19[79]:255-260. [DOI: 10.15557/ JoU. 2019.0038]. 